\title{
Adrenal Insufficiency, Sex Reversal, and Angelman Syndrome due to Uniparental Disomy Unmasking a Mutation in CYP11A1
}

\author{
Ahlee Kim Masanobu Fujimoto Vivian Hwa Philippe Backeljauw \\ Andrew Dauber \\ Division of Endocrinology, Cincinnati Children's Hospital Medical Center, Cincinnati, OH, USA
}

\section{Established Facts}

- Cholesterol side-chain cleavage enzyme deficiency (P450scc) is a rare genetic disorder that manifests with primary adrenal insufficiency with or without a disorder of sexual development (DSD).

- Autosomal recessive conditions can be unmasked by uniparental disomy (UPD).

\section{Novel Insights}

- We describe the first patient with Angelman syndrome due to a large region of segmental UPD and a mutation in CYP11A1, the gene that encodes P450scc, presenting with multiple phenotypes including developmental delay, seizures, primary adrenal insufficiency, and a DSD (testes with external female genitalia in a setting of a 47,XXY karyotype).

- Recent advancements in medical technology, especially in genetic diagnostics, have empowered us to identify clear genetic etiologies for conditions with previously unknown causes.

\section{Keywords}

CYP11A1 · P450 side-chain cleavage $\cdot$ Angelman syndrome $\cdot$ Primary adrenal insufficiency $\cdot$ Sex reversal $\cdot$ Genetics

\section{Abstract \\ Background/Aims: Cholesterol side-chain cleavage enzyme (P450scc) deficiency is a rare genetic disorder causing pri- mary adrenal insufficiency with or without a 46,XY disorder of sexual development (DSD). Herein, we report a case of the combination of primary adrenal insufficiency, a DSD (testes with female external genitalia in a setting of a 47, XXY karyo-}

\section{KARGER}

(c) 2018 S. Karger AG, Basel

E-Mail karger@karger.com

www.karger.com/hrp type), and Angelman syndrome. Methods: Comprehensive genetic analyses were performed, including a single nucleotide polymorphism microarray and whole-exome sequencing. In vitro studies were performed to evaluate the pathogenicity of the novel mutation that was identified by wholeexome sequencing. Results: The patient was found to have segmental uniparental disomy (UPD) of chromosome 15 explaining her diagnosis of Angelman syndrome. Wholeexome sequencing further revealed a novel homozygous intronic variant in CYP1 1A1, the gene encoding P450scc, found

Ahlee Kim and Masanobu Fujimoto contributed equally to this work.

Andrew Dauber, MD

Division of Endocrinology, Cincinnati Children's Hospital Medical Center 3333 Burnet Ave., MLC 7012

Cincinnati, OH 45229 (USA)

E-Mail Andrew.dauber@cchmc.org 
within the region of UPD. In vitro studies confirmed that this variant led to decreased efficiency of CYP11A1 splicing. Conclusion: We report the first case of the combination of 2 rare genetic disorders, Angelman syndrome, and P450scc deficiency. After 20 years of diagnostic efforts, significant advances in genetic diagnostic technology allowed us to determine that these 2 disorders originate from a unified genetic etiology, segmental UPD unmasking a novel recessive mutation in CYP11A1.

(c) 2018 S. Karger AG, Basel

\section{Introduction}

Angelman syndrome and P450 side-chain cleavage (P450scc) deficiency are 2 rare genetic disorders. Herein, we present the first case of a patient with these 2 disorders presenting with developmental delay, epilepsy, primary adrenal insufficiency, and a disorder of sexual development (DSD, normal testes with female external genitalia in a setting of a 47,XXY karyotype) due to a combination of segmental uniparental disomy (UPD) and a novel splicing variant in CYP11A1, the gene that encodes P450scc.

\section{Case Report}

A phenotypic female infant was born at a gestational age of 36 weeks to healthy nonconsanguineous parents. On initial examination, the infant was healthy with a birth weight of 2,949 $\mathrm{g}$ and length of $48.9 \mathrm{~cm}$. She had normal female external genitalia. At 3 months of age, she was noted to have developmental delay. Due to persistent developmental delay and hypotonia, she was evaluated by a pediatric neurologist at 13 months of age. A cytogenetic analysis revealed that she had a 47,XXY karyotype consistent with Klinefelter syndrome. Given the discordance between her chromosomal sex and external genitalia, a pelvic ultrasound was performed which failed to visualize mullerian structures or gonads. At 14 months of age, she was referred to pediatric endocrinology. Gonadotropin concentrations were FSH of $6 \mathrm{mIU} / \mathrm{mL}$ and LH less than $2.4 \mathrm{mIU} / \mathrm{L}$. Human chorionic gonadotropin (hCG) stimulation did not show any increase in testosterone (Table 1). Magnetic resonance imaging (MRI) of the abdomen failed to visualize gonads, and an MRI of the brain revealed mild cortical atrophy. At 18 months of age, she underwent exploratory laparoscopy which showed normal appearing bilateral testes at the external inguinal ring with apparently normal appearing wolffian structures, confirming the diagnosis of a DSD manifesting with testes and female external genitalia in the setting of a 47,XXY karyotype. For the sake of brevity, we will refer to her sexual development phenotype as "sex reversal." Further endocrine workup for the confirmed diagnosis of sex reversal showed mullerian-inhibiting substance of 22 $\mathrm{ng} / \mathrm{mL}$ (normal female reference $<3 \mathrm{ng} / \mathrm{mL}$ ). Based on both normal appearing gonads and the presence of mullerian-inhibiting substance, she underwent a repeat hCG stimulation study without an increase in testosterone concentration. To assess for the possibility of complete androgen insensitivity syndrome, although her hormonal phenotype was not typical for androgen insensitivity syndrome due to the lack of elevated androgens, androgen receptor sequencing was performed which was negative. At the age of 22 months, she underwent a bilateral gonadectomy, and the pathology reports described mildly hypoplastic testes with a confirmed karyotype of 47,XXY.

At 15 months of age, she demonstrated failure to thrive. Evaluation showed a low serum sodium $(131 \mathrm{mMol} / \mathrm{L})$ and a high potassium $(6.1 \mathrm{mMol} / \mathrm{L})$ in the absence of acidosis and hypoglycemia. With a high-dose adrenocorticotropic hormone (ACTH) (cosyntropin) stimulation test, baseline cortisol of $13.5 \mu \mathrm{g} / \mathrm{dL}$ increased only to $15.2 \mu \mathrm{g} / \mathrm{dL}$ after stimulation (Table 2). She was therefore diagnosed with primary adrenal insufficiency. At the age of 6 years, the diagnosis of primary adrenal insufficiency was confirmed based on an elevated ACTH of 5,000 pg/mL (normal range 5-27 $\mathrm{pg} / \mathrm{mL}$ ) and a low morning cortisol level of $3.0 \mu \mathrm{g} / \mathrm{dL}$ measured after omitting the prior evening's dose of hydrocortisone. Given the previous diagnosis of DSD and the lack of response to either hCG or ACTH stimulation, a common defect in both adrenal and gonadal steroidogenesis was speculated. She was started on hydrocortisone, fludrocortisone, and sodium chloride supplements which resulted in resolution of her electrolyte abnormalities and failure to thrive. At the age of 3 years, she was diagnosed with epilepsy after developing new-onset right-sided focal seizures and was started on anti-epileptic medication. She continued to have severe developmental delays of unexplained etiology.

At the age of 13 years, further diagnostic testing was performed in an attempt to identify a unifying diagnosis for the patient. Sequencing of the steroidogenic acute regulatory protein (StAR) gene, a known cause of primary adrenal insufficiency and sex reversal, was negative. A comparative genomic hybridization microarray was done which failed to detect any significant copy number changes other than the previously known 47,XXY karyotype. Subsequently, a methylation-specific polymerase chain reaction (PCR) analysis of the Prader-Willi/Angelman syndrome region was performed which revealed an absence of the maternal allele at 15q1113 consistent with Angelman syndrome. This test was unable to distinguish between a 15q deletion and UPD as a cause of the Angelman syndrome. This diagnosis explained her developmental delays and seizure disorder, but did not explain her adrenal insufficiency or sex reversal. Nine years after being diagnosed with Angelman syndrome, at the age of 22 years, she was evaluated by a multidisciplinary team in an attempt to identify a unifying genetic diagnosis.

\section{Materials and Methods}

\section{Genetic Analysis}

A single nucleotide polymorphism (SNP) microarray was performed using the Infinium Assay with the Illumina CytoXNP$850 \mathrm{Kv} 1.1$ BeadChip platform. This chip contains approximately 843,390 genome-wide markers, an overall average probe spacing of $1.8 \mathrm{~kb}$, and an average effective resolution of $18 \mathrm{~kb}$ to determine copy number change. Whole-exome sequencing was performed using previously described methods [1]. Sanger sequencing of the 
Table 1. Results of the hCG stimulation test

\begin{tabular}{lllll}
\hline & $\begin{array}{l}\text { Androstenedione, } \\
\mathrm{ng} / \mathrm{dL}\end{array}$ & $\begin{array}{l}\text { DHEA, } \\
\mathrm{ng} / \mathrm{dL}\end{array}$ & $\begin{array}{l}\text { Cortisol, } \\
\mu \mathrm{g} / \mathrm{dL}\end{array}$ & $\begin{array}{l}\text { Testosterone, } \\
\mathrm{ng} / \mathrm{mL}\end{array}$ \\
\hline Before stimulation & $8(5-32)$ & $8(9-113)$ & $2.9(6-25)$ & $<5$ \\
After stimulation & $7(5-32)$ & $8(9-113)$ & $3.6(6-25)$ & $<5$ \\
\hline
\end{tabular}

Table 2. Results of 2 high-dose ACTH stimulation tests

\begin{tabular}{|c|c|c|c|c|c|c|c|}
\hline & $\begin{array}{l}\text { Cortisol, } \\
\mu \mathrm{L} / \mathrm{dL}\end{array}$ & $\begin{array}{l}\text { Aldosterone, } \\
\mathrm{ng} / \mathrm{dL}\end{array}$ & $\begin{array}{l}\text { 17-OHP, } \\
\mathrm{ng} / \mathrm{mL}\end{array}$ & $\begin{array}{l}\text { Testosterone, } \\
\mathrm{ng} / \mathrm{mL}\end{array}$ & $\begin{array}{l}\text { Progesterone, } \\
\mathrm{pg} / \mathrm{mL}\end{array}$ & $\begin{array}{l}\text { Pregnenolone, } \\
\mathrm{ng} / \mathrm{dL}\end{array}$ & $\begin{array}{l}\text { 17-OH pregnenolone, } \\
\mathrm{ng} / \mathrm{dL}\end{array}$ \\
\hline \multicolumn{8}{|l|}{ First test } \\
\hline Before stimulation & $13.5(6-25)$ & $7.6(5-43)$ & & $<5$ & & $47(10-93)$ & $<10(14-207)$ \\
\hline After stimulation & $15.2(23-40)$ & $7.4(28-85)$ & & $<5$ & & & \\
\hline \multicolumn{8}{|l|}{ Second test } \\
\hline After stimulation & $16(23-40)$ & $16.7(28-85)$ & $197(65-353)$ & $<5$ & $<100(51-219)$ & & \\
\hline
\end{tabular}

specific CYP11A1 variant was performed in the subject and her parents. PCR primers are available on request. Written informed consent was obtained for research exome sequencing from the subject's legal guardian. The study was approved by the Cincinnati Children's Hospital Medical Center Institutional Review Board.

\section{CYP11A1 Minigene Construction}

Genomic DNAs of the family members and wild-type (WT) control were isolated from peripheral blood lymphocytes using PureLink Genomic DNA Mini Kit (Invitrogen, Carlsbad, CA, USA). PCR primers were designed to include the restriction sites Not I and EcoRI, for directional cloning into expression vector pcDNA3.1. The sequences of the primers were as follows: forward primer, 5 '-TTTGAATTCCCTTTGGGGCCATCAGCTTC-3' (EcoR1 site, underlined); reverse primer, 5'-TTTGCGGCCGCGATTGGTGCCTTCATTA-3' (NotI site, underlined). CYP11A1 genomic DNA consisting of exon 7, intron 7, and exon 8, was amplified by PCR using the above primers and GoTaq Flexi DNA Polymerase (Promega, Fitchburg, WI, USA). PCR was performed as follows: 1 cycle at $95^{\circ} \mathrm{C}$ for $2 \mathrm{~min}$, followed by 40 cycles $\left(95^{\circ} \mathrm{C}\right.$, $30 \mathrm{~s} ; 61^{\circ} \mathrm{C}, 30 \mathrm{~s}$; and $72^{\circ} \mathrm{C}, 60 \mathrm{~s}$ ) with a final extension of $72^{\circ} \mathrm{C}$ for $5 \mathrm{~min}$. The PCR products were electrophoresed on $2 \%$ agarose gels to confirm the expected amplification of the $869 \mathrm{bp}$ products. The PCR products were digested with NotI and EcoRI (New England Biolabs, Ipswich, MA, USA), $37^{\circ} \mathrm{C}$ for $60 \mathrm{~min}$, directionally cloned (T4 DNA ligase, New England Biolabs) into pcDNA3.1(+) plasmid (Invitrogen), and transformed into Subcloning Efficiency DH5a Competent Cells (Invitrogen). Ampicillin $(1: 1,000)$ resistance colonies were screened for plasmids carrying the 869 bp inserts (plasmid isolated using QIAprep Spin Miniprep Kit, QIAGEN, Hilden, Germany). The sequences of the inserts were confirmed by Sanger sequencing with the same primers. We used NCBI reference sequence: NG_007973.1. for the genomic reference and NM_000781.2 for the mRNA reference to confirm the sequence.

P450scc Deficiency in Angelman Syndrome

\section{CYP11A1 Minigene Splicing Analysis}

Three each of WT and mutant minigene constructs were selected for the splicing assay [2]. HEK293 cells were plated in 6-well plates and cultured in DMEM containing 10\% fetal bovine serum at $37^{\circ} \mathrm{C}$ in $5 \% \mathrm{CO}_{2}$. The cells at $70-80 \%$ confluency were transfected with the WT or mutant variant construct $(1 \mu \mathrm{g} /$ well $)$ using $3 \mu \mathrm{L}$ of PolyJet In Vitro DNA Transfection Reagent (SignaGen Laboratories, Rockville, MD, USA) according to the manufacturer's recommended protocol. The culture media was replaced $5 \mathrm{~h}$ after transfection. The cells were cultured for an additional $24 \mathrm{~h}$ and total RNA was extracted using RNeasy Mini (QIAGEN, Hilden, Germany) according to the manufacturer's instructions. RNA samples were reverse transcribed (SuperScript IV FirstStrand Synthesis System, Invitrogen), following the manufacturer's instructions, and PCR amplified as described above. PCR products were fractionated on $2 \%$ agarose gels, stained with ethidium bromide, and visualized. Each DNA fragments on the band in the gels were extracted using Zymoclean Gel DNA Recovery Kit (Zymo Research, Irvine, CA, USA) and their sequence was confirmed by Sanger sequencing.

\section{Results}

\section{Genetic Analysis}

An SNP microarray confirmed the lack of significant genomic copy number variants but revealed a 78.0 $\mathrm{Mb}$ region of homozygosity extending from $15 q 11.1$ to $15 q 26.2$ consistent with segmental UPD. Taken together with her prior testing for Angelman syndrome, this finding confirmed that her diagnosis of Angelman syndrome was caused by paternal UPD of the Angelman region. In addition, the large size of the region of homozygosity raised the 


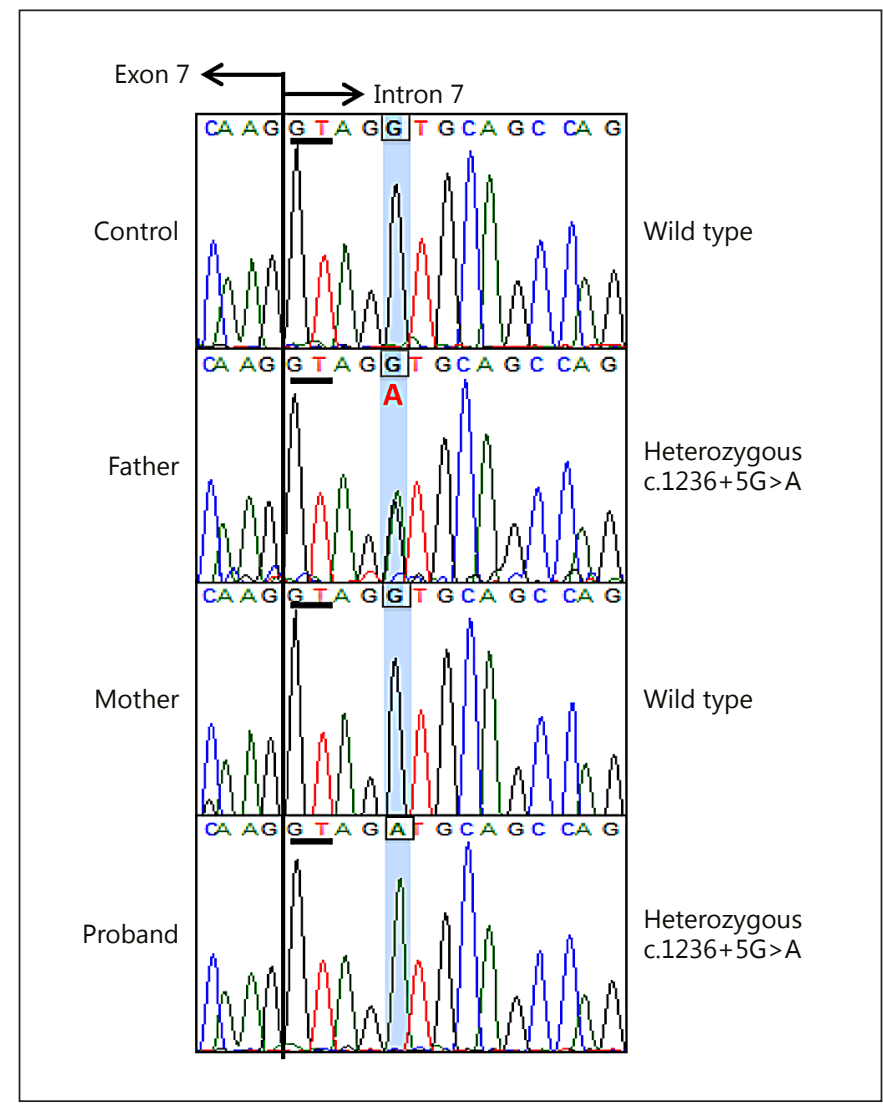

Fig. 1. Electropherograms showing DNA sequences of the CYP11A1 exon 7-intron 7 junctions in the affected family. Sequences of family members, compared to wild type, indicated homozygosity of c. $1236+5 \mathrm{G}>\mathrm{A}$ in the proband, which confirmed the uniparental transmission from the father who was the heterozygous carrier. The underlined GT indicates the intronic invariant dinucleotides of the donor splice site.

possibility of a potential autosomal recessive disorder in this region. There were a total of 62 recessive disease-causing genes in the homozygous region. One of those was CYP11A1, a rare known cause of primary adrenal insufficiency and sex reversal. To assess for the possibility of a mutation in CYP11A1 and to rule out any other recessive genetic condition unmasked by the segmental UPD, we performed whole-exome sequencing. There were no novel nonsynonymous homozygous variants found within the region of UPD. However, we did identify a novel homozygous variant 5 bases into intron 7 of CYP11A1 (c.1236+5G>A, chr15:74631573 C>T) (Fig. 1). DNA sequencing of the family members identified that the father is a heterozygous carrier and the mother is WT, confirming a paternal uniparental transmission (Fig. 1).

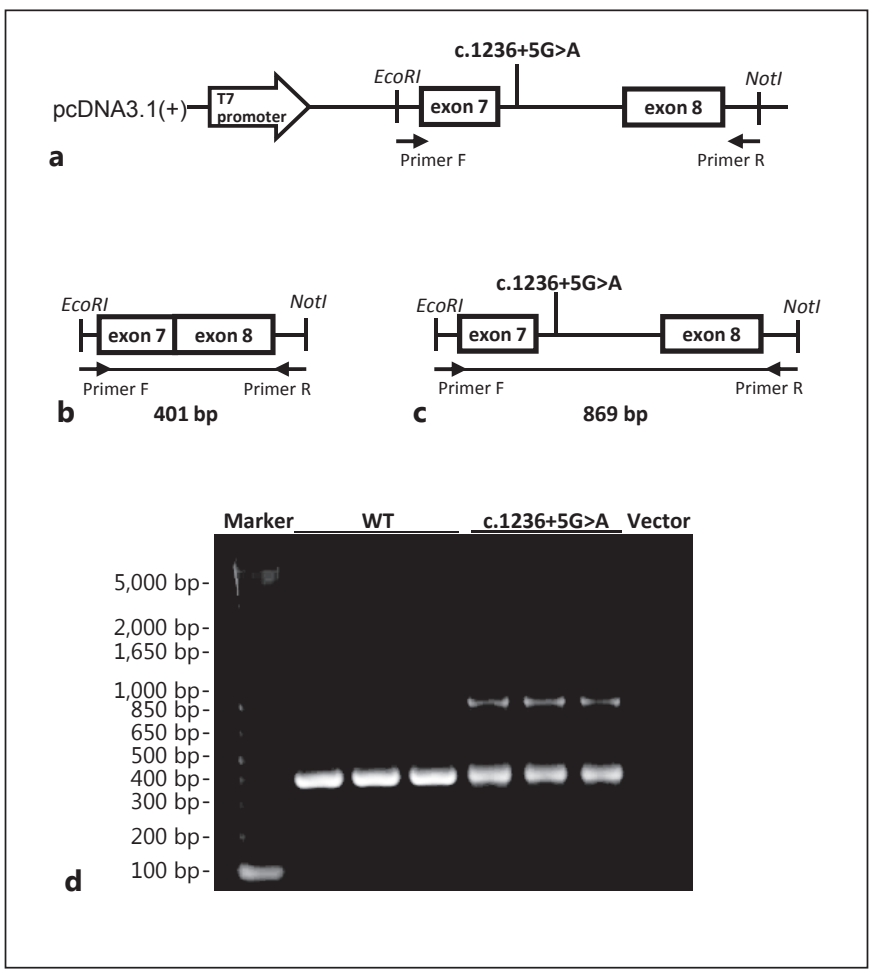

Fig. 2. CYP11A1 minigene splicing analysis. a Schema of the CYP11A1 minigene. The vertical bars show the site of the variant residue in intron 7 (c.1236+5G $>A$ ) and the recognition sites for the restriction enzymes. The arrows show the positions of the primers used in PCR and RT-PCR. b Schema of the correctly spliced mRNA fragment. The expected length of the RT-PCR product is 401 bp. c Schema of the mis-spliced mRNA fragment with the retained intron 7. The expected length of the RT-PCR product is 869 bp. d Agarose gel electrophoretic pattern of the PCR products. The PCR products from 3 clones each for (wild type) WT and mutant variant were tested. The PCR products of WT show the expected $401 \mathrm{bp}$ splice product from the WT construct. The PCR products of the mutant variant show the expected $401 \mathrm{bp}$ and the misspliced 869 bp from the mutant variant construct indicating that the mutant variant leads to decreased splicing efficiency. Each of the DNA bands in the gel was extracted and these sequences were confirmed by Sanger sequencing.

\section{CYP11A1 Minigene Splicing Analysis}

Given the proximity of this variant to the exon/intron boundary, we speculated that the mutation may disrupt normal excision of the CYP11A1 intron 7. To test our hypothesis, we performed an in vitro minigene splicing assay. The c. $1236+5 \mathrm{G}>\mathrm{A}$ variant led to decreased efficiency of splicing out of intron 7 resulting in the presence of an unspliced product containing exons 7 and 8 with the retained intervening intron 7 (Fig. 2). This result supports the pathogenicity of this specific variant. 


\section{Discussion}

We report the first case of Angelman syndrome, primary adrenal insufficiency, and sex reversal due to segmental UPD unmasking a novel recessive mutation in CYP11A1 in a $47, \mathrm{XXY}$ patient. P450scc deficiency is a rare disorder that leads to primary adrenal insufficiency with or without sex reversal. P450scc is required to catalyze the conversion of cholesterol to pregnenolone, the first and rate-limiting step of steroid hormone synthesis following mitochondrial cholesterol import by StAR [3]. Initially, P450scc deficiency was considered to be fatal in utero as the enzyme is required to produce placental progesterone, a critical hormone to maintain a normal pregnancy. However, a patient born at term with primary adrenal insufficiency and sex reversal due to a definitive P450scc defect was first described in 2005 [4]. Since then, more than 28 patients with $\mathrm{P} 450 \mathrm{scc}$ defect have been reported with various degrees of clinical severity and enzyme activity impairment $[4,5]$. Our patient likely has partial P450scc deficiency caused by the novel homozygous splicing mutation in CYP11A1. It is intriguing that in our patient, $\mathrm{P} 450 \mathrm{scc}$ deficiency was unmasked by segmental UPD of chromosome 5 (15q11.1 to 15q26.2), which also caused Angelman syndrome. Angelman syndrome, a neurodevelopmental disorder first described in 1965 , is caused by 4 known genetic mechanisms including molecular deletions involving the 15q11.2-q13, paternal UPD, imprinting defects, and mutations in the ubiquitin-protein ligase E3A gene (UBE3A) [6]. Patients with Angelman syndrome present with developmental delay, ataxia, and seizures. The prevalence of this condition is about 1 in 10,000 to 1 in 20,000 [7]. To our knowledge, an association between these 2 rare disorders has not been reported. The fact that our patient also has Klinefelter syndrome is possibly just a coincidence given how common Klinefelter syndrome is in the general population [8].

It is interesting that our patient presented with quite a significant phenotype of disordered sexual development as compared to her late presentation with primary adrenal insufficiency. Her adrenal phenotype was relatively mild for a patient with CYP11A1 deficiency which may be due to her having a partial loss of function from the homozygous splicing variant. As she is the first patient reported with a homozygous splicing defect, it is difficult to definitively conclude whether other patients with splicing defects will have a similar phenotype. We speculate that the etiology for her disordered sexual development may be multifactorial, including the coexisting Klinefelter syndrome. While the majority of individuals with a 47,XXY karyotype have normal male external genitalia, variable DSD phenotypes have been uncommonly observed in Klinefelter patients [9]. These phenotypes can vary widely from complete sex reversal or complete androgen insensitivity to mild abnormalities, such as micropenis, hypospadias, or undescended testes. The mechanisms of DSD in the setting of Klinefelter syndrome have been associated with variable androgen production within the critical period of sex development in utero, insufficient androgen action due to the CAGn trinucleotide repeat polymorphism of the androgen receptor gene, or the effect from extra copy of the DAX-1 gene $[9,10]$. On review of the exome data, our patient had a CAG repeat length of 22 which is in the moderate range. This alone is unlikely to account for her genital phenotype, but it may be a contributing factor in combination with the CYP11A1 deficiency.

The complexity of our patient's clinical presentation based on these 2 rare genetic disorders led to a diagnostic odyssey that lasted over 20 years. At the time of the patient's initial presentation, defects in P450scc had not been described, and the initial testing for Angelman syndrome was unable to distinguish between a genomic deletion and UPD. With recent technological advances in medical genetics, physicians now have a much greater capacity for unraveling these diagnostic dilemmas. We utilized a combination of an SNP array, exome sequencing, and an in vitro splicing assay to identify the genetic etiology in our patient to provide an explanation for this patient's complete clinical picture of developmental delay, epilepsy, adrenal insufficiency, and sex reversal. This case highlights the risk of UPD unmasking a recessive genetic condition and should raise awareness of the possibility of a CYP11A1 defect in patients with Angelman syndrome who present with adrenal insufficiency with or without sex reversal.

\section{Disclosure Statement}

The authors have no conflicts of interest to disclose.

\section{Funding Sources}

This work was supported by grant K23HD07335 (to A.D.) from the Eunice Kennedy Shriver National Institute of Child Health and Human Development of the National Institutes of Health (NIH). Additional support was provided by the National Center for Advancing Translational Sciences, NIH, through Grant 1UL1 TR001425. The content is solely the responsibility of the authors and does not necessarily represent the official views of the NIH. 


\section{References}

1 de Bruin C, Mericq V, Andrew SF, van Duyvenvoorde HA, Verkaik NS, Losekoot M et al. An XRCC4 splice mutation associated with severe short stature, gonadal failure, and early-onset metabolic syndrome. J Clin Endocrinol Metab. 2015 May;100(5):E789-98.

2 Kim CJ, Lin L, Huang N, Quigley CA, AvRuskin TW, Achermann JC et al. Severe combined adrenal and gonadal deficiency caused by novel mutations in the cholesterol side chain cleavage enzyme, P450scc. J Clin Endocrinol Metab. 2008 Mar;93(3):696-702.

3 Shikita M, Hall PF. Cytochrome P-450 from bovine adrenocortical mitochondria: an enzyme for the side chain cleavage of cholesterol. II. Subunit structure. J Biol Chem. 1973 Aug;248(16):5605-9.
4 Hiort O, Holterhus PM, Werner R, Marschke C, Hoppe U, Partsch CJ et al. Homozygous disruption of P450 side-chain cleavage (CY$\mathrm{P} 11 \mathrm{~A} 1)$ is associated with prematurity, complete 46,XY sex reversal, and severe adrenal failure. J Clin Endocrinol Metab. 2005 Jan; 90(1):538-41.

5 Miller WL. Disorders in the initial steps of steroid hormone synthesis. J Steroid Biochem Mol Biol. 2017 Jan;165(Pt A):18-37.

6 Williams CA, Beaudet AL, Clayton-Smith J, Knoll JH, Kyllerman M, Laan LA et al. Angelman syndrome 2005: updated consensus for diagnostic criteria. Am J Med Genet A. 2006 Mar;140(5):413-8.
7 Tan WH, Bird LM. Angelman syndrome: current and emerging therapies in 2016. Am J Med Genet C Semin Med Genet. 2016 Dec; 172(4):384-401.

8 Visootsak J, Graham JM Jr. Klinefelter syndrome and other sex chromosomal aneuploidies. Orphanet J Rare Dis. 2006 Oct;1(1):42.

9 Lee YS, Cheng AW, Ahmed SF, Shaw NJ, Hughes IA. Genital anomalies in Klinefelter's syndrome. Horm Res. 2007;68(3):150-5.

10 Zitzmann M, Depenbusch M, Gromoll J, Nieschlag E. X-chromosome inactivation patterns and androgen receptor functionality influence phenotype and social characteristics as well as pharmacogenetics of testosterone therapy in Klinefelter patients. J Clin Endocrinol Metab. 2004 Dec;89(12):6208-17. 